\title{
Pressure-Driven Metal-Insulator Transition in Hematite from Dynamical Mean-Field Theory
}

\author{
J. Kuneš, ${ }^{1}$ Dm. M. Korotin, ${ }^{2}$ M. A. Korotin,${ }^{2}$ V. I. Anisimov, ${ }^{2}$ and P. Werner ${ }^{3}$ \\ ${ }^{1}$ Theoretical Physics III, Center for Electronic Correlations and Magnetism, \\ Institute of Physics, University of Augsburg, Augsburg 86135, Germany \\ ${ }^{2}$ Institute of Metal Physics, Russian Academy of Sciences, 620041 Yekaterinburg GSP-170, Russia \\ ${ }^{3}$ Theoretische Physik, ETH Zurich, 8093 Zurich, Switzerland
}

\begin{abstract}
The Local Density Approximation combined with Dynamical Mean-Field Theory (LDA+DMFT method) is applied to the study of the paramagnetic and magnetically ordered phases of hematite $\mathrm{Fe}_{2} \mathrm{O}_{3}$ as a function of volume. As the volume is decreased, a simultaneous 1st order insulatormetal and high-spin to low-spin transition occurs close to the experimental value of the critical volume. The high-spin insulating phase is destroyed by a progressive reduction of the charge gap with increasing pressure, upon closing of which the high spin phase becomes unstable. We conclude that the transition in $\mathrm{Fe}_{2} \mathrm{O}_{3}$ at $\approx 50 \mathrm{GPa}$ can be described as an electronically driven volume collapse.

PACS numbers: 74.25.Jb, 71.27.+a, 71.30.+h, 91.60.Gf
\end{abstract}

Metal-insulator transition (MIT) is one of the central topics in the physics of strongly correlated electron materials. Despite considerable progress in the theoretical understanding of this phenomenon in the past decade, we are only starting to uncover the multitude of possible transition scenarios in real materials. An important instance of the MIT is the pressure driven transition accompanied by a change of the local spin state (high spin (HS) to low spin (LS) transition) seen in $\mathrm{MnO}$ [1], $\mathrm{BiFeO}_{3}$ 2] or $\mathrm{Fe}_{2} \mathrm{O}_{3}$. The understanding of the pressure-driven HS-LS transition and its relationship to eventual MIT and structural and/or volume changes is relevant to a broader class of oxides - often with important geological implications - such as $\mathrm{FeO}, \mathrm{CoO}$ or $\mathrm{Fe}_{3} \mathrm{O}_{4}$.

In this Letter we study the spin and metal-insulator transition in hematite $\left(\alpha-\mathrm{Fe}_{2} \mathrm{O}_{3}\right)$ under pressure using the numerical LDA+DMFT approach [3]. At ambient conditions, hematite is an antiferromagnetic insulator $\left(T_{N}=956 \mathrm{~K}\right)$ with the corundum structure [4]. The iron ions having a formal $\mathrm{Fe}^{3+}$ valency with five $d$ electrons give rise to a local HS state. Photoemission studies [5, 6] classified hematite as charge-transfer insulator. A gap of 2.0-2.7 eV was inferred from the electrical conductivity data [7]. Under pressure, a first-order phase transition is observed at approximately $50 \mathrm{GPa}$ ( $82 \%$ of the equilibrium volume) at which the specific volume decreases by almost $10 \%$ and the crystal-lattice symmetry is reduced (a structure of the $\mathrm{Rh}_{2} \mathrm{O}_{3}$-II type is formed) [8, 9, , 10]. The high-pressure phase is characterized by a metallic conductivity and absence of both the long-range magnetic order and the HS local moment [9]. Badro et al. showed that the structural transition actually precedes the electronic transition, which is nevertheless accompanied by a sizable reduction of the bond lengths [11].

The investigations of the electronic structure of hematite using various theoretical approaches produced different (and frequently controversial) results. Similar to many transition metal oxides, calculations within the local spin density (LSDA) and generalized gradient (GGA) approximations failed to reproduce the ground state characteristics under ambient conditions, underestimating values of the magnetic moment of iron and of the energy gap [12]. Within GGA, the structural transition together with a transition into the metallic ferromagnetic state with $\mathrm{Fe}$ ions in the LS state was predicted at a pressure of $14 \mathrm{GPa}$ [13], which is about one fourth of the experimental value. The studies using the LSDA+U approach, which treats the local Coulomb interaction on the static mean-field level 13] lead to improved values of the magnetic moment and the gap in the HS phase, but failed to capture the metal-insulator transition. Like for some other oxides, LSDA+U improves the description of the HS low-pressure phase, while LSDA appears more appropriate for the description of the LS high-pressure phase, none of them covering the full pressure range. Thus, the DMFT method, which can capture the evolution from strong to weak local correlations is a promising tool for investigating the physics in the transition regime, as was shown for $\mathrm{MnO}$ [14]. The only available DMFT study of $\mathrm{Fe}_{2} \mathrm{O}_{3}$ to-date was limited to the $t_{2 g}$ band manifold finding a low-spin metallic phase at high pressure [15].

In this work we use a Hamiltonian including explicitly all O- $p$ and Fe- $d$ orbitals, which allows us to address the following questions. i) Is there an electronic transition without invoking a change in crystal structure? ii) Is there a simultaneous metal-insulator and local moment transition as found in the isoelectronic $\mathrm{MnO}$ [14] iii) What is the nature of the transition and how is it affected by temperature and long-range magnetic order? We use an implementation of LDA+DMFT, previously applied to $\mathrm{NiO}$ and described in Ref. 16, which combines the first-principles multi-band Hubbard Hamiltonian

$$
\hat{H}=\sum_{k} \hat{\mathbf{a}}_{k}^{\dagger} \mathbf{H}_{k} \hat{\mathbf{a}}_{k}-\varepsilon_{d c}\left(n_{d}\right) \hat{N}_{d}+\sum_{i \in F e} \hat{\mathbf{n}}_{i}^{d} \mathbf{U}^{d d} \hat{\mathbf{n}}_{i}^{d}
$$

with DMFT equations. Here, $\mathbf{H}_{k}$ is the LDA Hamil- 


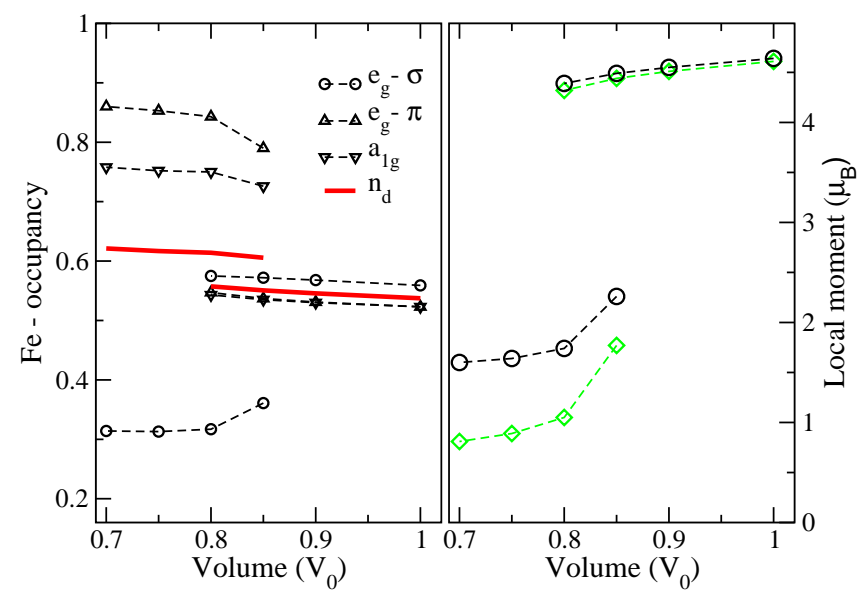

FIG. 1: Orbital occupancies (left panel) at various specific volumes in $\mathrm{PM}$ state at $\mathrm{T}=580 \mathrm{~K}$. The thick line marks the average $d$ occupancy per orbital. Corresponding values of the screened (diamonds) and instantaneous (circle) local moment are shown in the right panel.

tonian matrix $\left(38 \times 38\right.$ plus spin degeneracy; two $\mathrm{Fe}_{2} \mathrm{O}_{3}$ units) constructed on the basis of Fe- $d$ and O- $p$ Wannier orbitals represented numerically on a uniform kmesh in the first Brillouin zone [17]. The second term is the double-counting correction amounting to a constant shift applied to Fe- $d$ site energies. The last term is the two-particle interaction at the Fe sites in the densitydensity approximation, parameterized with $U=6.8 \mathrm{eV}$ and $J=0.86 \mathrm{eV}[18]$. (Fitting the experimental photoemission spectra yields $\mathrm{U}$ of $8 \mathrm{eV}$ according to Ref. 5 or $7 \mathrm{eV}$ according to Ref. 19.) For the double-counting correction $\varepsilon_{d c}$ we have adopted the prescription $\varepsilon_{d c}=$ $\left(N_{\text {orb }}-1\right) \bar{U} \bar{n}_{d}$ of Ref. 16, but taking the self-consistent DMFT value for the average occupancy $\bar{n}_{d}$ rather than its LDA value. This approach, which can be viewed as a poor man's charge self-consistency loop, is necessary for stabilization of the ambient pressure phase as discussed later. For the solution of the $d$-shell impurity problem we have employed a continuous time quantum Mote-Carlo (CT-QMC) solver [20]. The calculations were performed in the corundum $\alpha-\mathrm{Fe}_{2} \mathrm{O}_{3}$ structure with rhombohedral (space-group R $\overline{3} \mathrm{c}$ ) unit cell containing two formula units. Throughout the paper the compressed phase is characterized by the volume relative to the experimental ambientpressure structure with lattice parameter $\mathrm{a}_{0}=5.361 \AA$, rhombohedral angle $\alpha=55^{\circ} 17^{\prime}$, and atomic coordinates $\mathrm{Fe}(0,0,0.355)$ and $\mathrm{O}(0.300,0,0.25)$. Volume reduction is achieved by scaling the lattice constant.

In Fig. 1 we show the evolution of the orbital occupancies and Fe local moment under compression in the absence of long-range magnetic order. The local moment is measured as the square root of i) $\left\langle m_{z}^{2}\right\rangle$ (instantaneous moment) or ii) $\frac{1}{\beta} \int_{0}^{\beta} d \tau\left\langle m_{z}(\tau) m_{z}(0)\right\rangle$ (screened moment), where $\beta$ is the inverse temperature. At the ambient con-

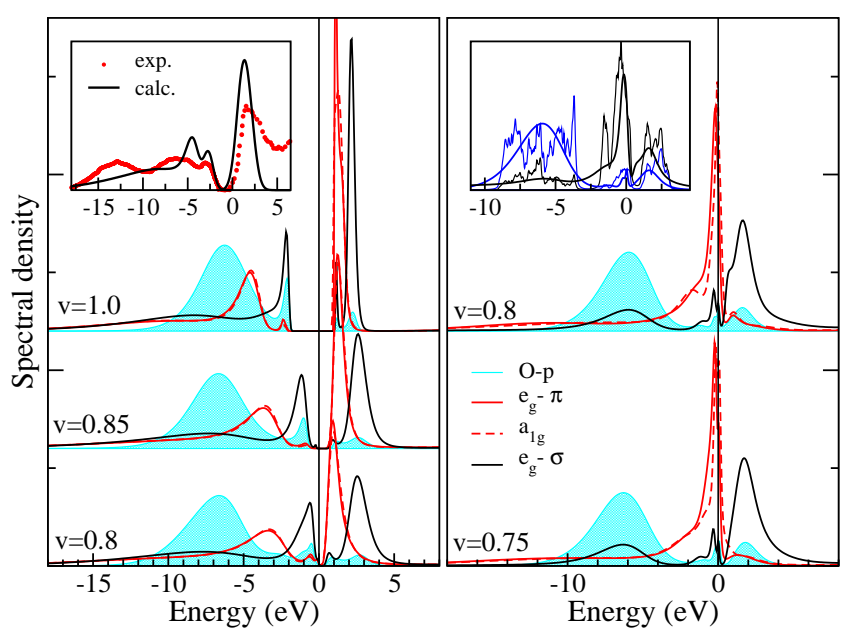

FIG. 2: (Color online) Evolution of the single-particle spectra with pressure obtain in the $\mathrm{PM}$ state at $\mathrm{T}=580 \mathrm{~K}$. The HS solutions are shown in the left and LS solutions in the right panel. All curves are normalized to one. The left inset presents comparison of experimental PES [6] and IPS [22] data (points) to the calculated Lorentzian broadened $d$ spectral density (solid line). The right inset shows a comparison of the $\mathrm{v}=0.8 \mathrm{DMFT}$ (smooth lines) and non-interacting LDA spectra (jagged lines): total $d$ spectral density (dark; black), total O- $p$ spectral density (light; blue).

ditions (volume $V_{0}$ ) we find a HS solution with both definitions of local moment yielding essentially the value of $4.6 \mu_{B}$. Upon compression the HS phase survives down to $0.8 V_{0}$. Below this volume only a LS phase with substantially reduced local moment is stable. The difference between its screened and instantaneous values indicates that the LS moment cannot be viewed a rigid object. The HS and LS solutions coexist in the range $0.8-0.85 V_{0}$. The Fe- $d$ orbital occupancies fit into the ionic picture of a fully spin-polarized $d$-shell in the HS state, and an orbitally polarized $d$-shell in the LS state. Nevertheless, the substantial $e_{g}^{\sigma}$ population in the LS phase reflects a sizable covalent $p-d$ bonding (hybridization between $\mathrm{O}-p$ and $\mathrm{Fe}-e_{g}$ orbitals).

In Fig. 2 we show the corresponding single-particle spectral densities. Note the difference of the O- $p$ spectral weight above the chemical potential between HS and LS solutions. In the LS state the $e_{g}^{\sigma}$-derived bands are above the chemical potential, carrying some admixture of O- $p$ character with them, which leads a charge transfer from the O- $p$ orbitals and an increase of $\bar{n}_{d}$ (see Fig. 1). Relaxation of the electronic states, which compensates this charge redistribution in real material, is mimicked by the self-consistency of the double counting term in the present work. Comparison to the photoemission and inverse photoemission data (see left inset of Fig. 2) reveals a fair agreement between the theory and experiment. The computed ambient pressure HS phase is found to be an insulator with a charge gap of $2.6 \mathrm{eV}$. Upon com- 


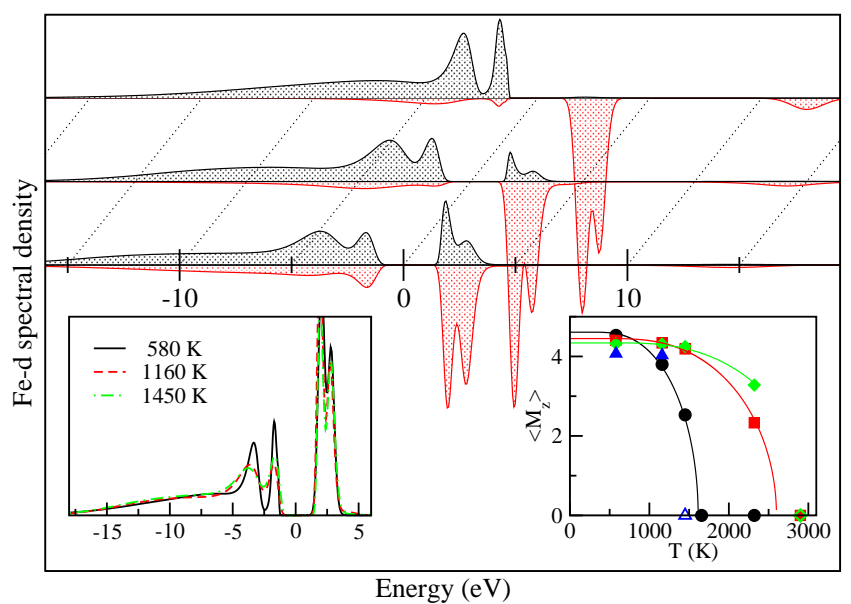

FIG. 3: Spin-polarized spectral Fe- $d$ spectral density at ambient pressure for $580 \mathrm{~K}, 1160 \mathrm{~K}$ and $1450 \mathrm{~K}$ from the top to the bottom. The left inset compares the same densities averaged over spin. The right inset shows the staggered magnetization vs temperature curves for $\mathrm{v}=1.0$ (circles; black), $\mathrm{v}=0.9$ (squares; red), v=0.85 (diamonds; green), and $\mathrm{v}=0.8$ (triangles; blue). At empty symbols only the LS solution exists. The lines depict mean-field fits.

pression the gap gradually shrinks due to broadening of the bands and increasing of crystal-field splitting. At the high-pressure end of the HS stability region $\left(0.8 V_{0}\right)$ the gap is reduced to a fraction of an $\mathrm{eV}$. The spectral density in the LS phase closely resembles the non-interacting one (see right inset of Fig. 2). Unlike in the HS phase, there is some orbital polarization within the $t_{2 g}\left(=a_{1 g}+e_{g}^{\pi}\right)$ orbitals, split due to distortion of $\mathrm{FeO}_{6}$ octahedra.

Next, we address the role of magnetic long-range order. Unlike LDA or LDA $+\mathrm{U}$, for which static magnetic order is essential to capture a HS insulator, in the present approach magnetic ordering can take place independently from the gap and local moment formation, as observed in many materials. Using a symmetry that allows for the experimental AFM order we observe the following behavior. At room temperature the HS solutions are magnetically ordered, while all LS solution remain paramagnetic. In Fig. 3 we show the spin-polarized Fe- $d$ spectral densities at several temperatures. Clearly, the charge gap and the main spectral features are insensitive to the presence of AFM order. The magnetization curves at reduced volume reflect the increase of the exchange coupling with shortened atomic distances.

Our observations are summarized in the phase diagram shown in Fig. 4. We distinguish three phases: i) paramagnetic LS, ii) antiferromagnetic HS and iii) paramagnetic HS, separated by the line of Néel temperature $T_{N}$ and the coexistence region of the HS and LS phases. The evaluation of $T_{N}$ is affected by two approximations, both favoring the ordered phase. First, the lack of nonlocal fluctuations inherent to DMFT and second, the approximate form of the local Coulomb interaction, limited

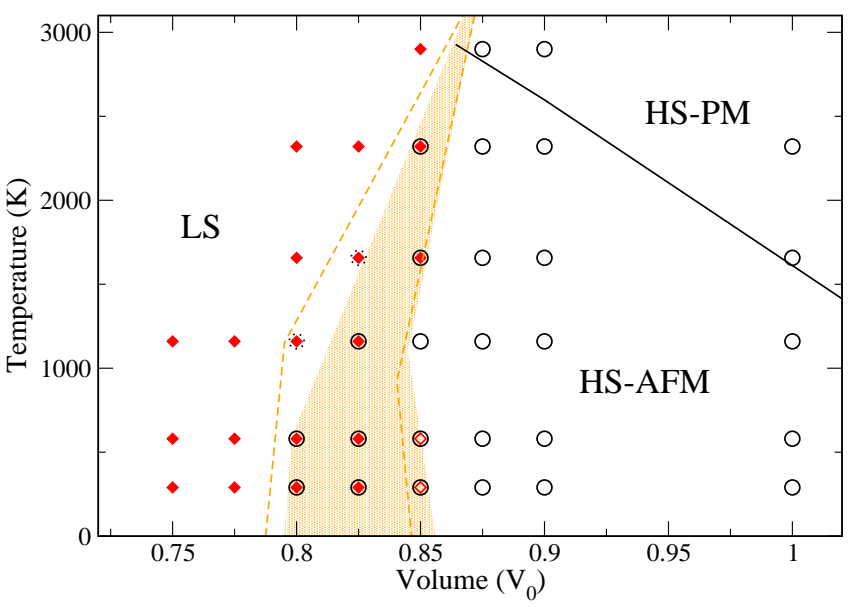

FIG. 4: Calculated V-T phase diagram of hematite. The symbols mark the actual computation results: HS state (circle), LS state (diamond). Empty diamonds mark LS solutions stable only if PM symmetry is constrained, dotted circles mark HS solutions stable only if AFM order is allowed. The black line shows the Néel temperature estimated from fits to $\mathrm{v}=1.0,0.9$ and 0.85 data. The shaded area marks the estimated HS/LS coexistence region with PM constraint, the dashed lines mark the coexistence regime when AFM order is allowed.

to Ising terms, thus rendering the HS local state two$\left(S_{z}= \pm 5 / 2\right)$ instead of sixfold degenerate $(S=5 / 2)$. The latter approximation is also reflected in the shape of the magnetization curves, which can be well fitted with a mean-field theory based on the Brillouin function $B_{1 / 2}$ (see the inset of Fig. 3). Given these approximations the calculated $T_{N}$ of $1600 \mathrm{~K}$ appears to be in reasonable agreement with the experimental value of $956 \mathrm{~K}$.

The effect of AFM order on the HS-LS transition is to slightly shift the boundaries of the coexistence region in favor of the HS phase. In particular, at the lowest studied temperatures we find the LS solution at $0.85 V_{0}$ to be unstable when AFM order is allowed. Also we find the HS solutions at $\left(0.8 V_{0}, 1160 \mathrm{~K}\right)$ and $\left(0.825 V_{0}, 1660 \mathrm{~K}\right)$ stabilized by AFM order. Interestingly, in the vicinity of the HS/LS boundary we find the magnetic order with increasing temperature not to be destroyed by magnetic fluctuations, but via a collapse of the constituting moments.

We propose two scenarios how the HS state can be destabilized and discuss them in the context of the present results, the $\mathrm{MnO}$ results of Ref. 14 and the model calculations of Ref. 21. We start with a simple estimate of the charge gap considering the ionic energies

$$
E_{\mathrm{g}} \approx E\left(d^{6}\right)+E\left(d^{4}\right)-2 E\left(d^{5}\right)-W=U+4 J-2 \Delta-W,
$$

where $2 \Delta$ stands for the difference of $t_{2 g}\left(=a_{1 g}+e_{g}^{\pi}\right)$ and $e_{g}^{\sigma}$ energies (as in Ref. 21), $W$ for the average of the $e_{g}$ and $t_{2 g}$ bandwidths, $E\left(d^{5}\right)$ is the energy of the HS ionic groundstate while $E\left(d^{6}\right)$ and $E\left(d^{4}\right)$ the ionic en- 
ergies of the state with one more and one less electron, respectively. Similarly one can compare the energies of the ionic HS and LS states to get

$$
E(L S)-E(H S)=10 J-4 \Delta .
$$

The HS state becomes unstable when either (1) or (2) becomes negative. Thus depending on the parameters the HS state can be destroyed due to gap closing or due to a local state transition. In charge-transfer compounds such as hematite $E_{\mathrm{g}}$ is substantially reduced by the presence of the ligand bands. We found that a similar simple estimate for a 2-band model provides a fairly accurate description of the phase boundaries found in Ref. 21.

Comparing the present results with the earlier study of isoelectronic $\mathrm{MnO}$ [14] there is an obvious similarity in that the HS phase is an insulator while the LS phase is metallic. However, there are two important differences in how the transition happens. First, in $\mathrm{MnO}$ the transition is found to proceed through a continuous sequence of intermediate states while in hematite the transition is first order with a coexistence region of HS and LS solutions. Second, we find the gap to almost close at the high-pressure end of the $\mathrm{HS}$ stability region in $\mathrm{Fe}_{2} \mathrm{O}_{3}$ while in $\mathrm{MnO}$ the transition starts with the appearance of an in-gap spectral density. The latter observation leads us to suggest that the HS-LS/insulator-metal transition in hematite proceeds through the gap closing mechanism while in $\mathrm{MnO}$ a local state transition takes place as was argued in Ref. 14. The gap closing picture of the transition in hematite is also supported by the shape of the HS/LS phase boundary, showing that HS state can be destroyed by thermal fluctuations, contrary to the local state transition picture where the HS state is expected to be favored at high temperature due to its higher entropy.

Finally, we briefly address the reported structure and volume changes $[9,10,11]$. The corundum and $\mathrm{Rh}_{2} \mathrm{O}_{3}-\mathrm{II}$ structures have similar local Fe environment and transitions between the two were reported in other materials without significant changes of electronic structure. In this study we do not attempt to distinguish between the two structural types. Finding the electronic HSLS/insulator-metal transition in the high-volume phase shows that the electronic transition is not implicated by the structural transition or volume collapse. Conversely the electronic transition provides a natural explanation of the volume collapse as a consequence of emptying of the anti-bonding $e_{g}^{\sigma}$ bands in the LS phase and corresponding strengthening of the Fe-O bonds.

In summary, we have observed a simultaneous HSLS/insulator-metal transition in the corundum phase of hematite close to the experimental volume in the numerical results obtained by LDA+DMFT. The long-range magnetic order is found to play a minor role in the transition. We have discussed two possible scenarios, the gap closing and the local state transition, and concluded that the transition in hematite, unlike in $\mathrm{MnO}$, proceeds through the gap closing mechanism. Our results are consistent with the picture of an electronically driven volume collapse.

J.K. acknowledges the support of SFB 484 of the Deutsche Forschungsgemeinschaft. Support by the Russian Foundation for Basic Research (project No. RFFI07-02-00041) and Presidium of the Russian Academy of Sciences (project No. 31, subprogram No. 3, Program No. 9) is gratefully acknowledged.

[1] C. S. Yoo et al., Phys. Rev. Lett. 94, 115502 (2005).

[2] A. G. Gavriliuk et al., Phys. Rev. B 77, 155112 (2008).

[3] K. Held, I. A. Nekrasov, G. Keller, V. Eyert, N. Blümer, A. K. McMahan, R. T. Scalettar, T. Pruschke, V. I. Anisimov, and D. Vollhardt, phys. stat. sol. (b) 243, 2599 (2006); G. Kotliar, S. Y. Savrasov, K. Haule, V. S. Oudovenko, O. Parcollet, C. A. Marianetti, Rev. Mod. Phys. 78, 865 (2006).

[4] C. G. Shull, W. A. Strauser, and E. O. Wollan, Phys. Rev. 83, 333 (1951).

[5] A. Fujimori, M. Saeki, N. Kimizuka, M. Taniguchi and S. Suga, Phys. Rev. B 34, 7318 (1986); C.-Y. Kim, M. J. Bedzyk, E. J. Nelson, J. C. Woicik, L. E. Berman, Phys. Rev. B 66, 085115 (2002).

[6] R. J. Lad and V. E. Henrich, Phys. Rev. B 39, 13478 (1989).

[7] S.Mochizuki, Phys.Status Solidi A 41, 591 (1977); K.H.Kim, S.-H. Lee and J.-S. Choi, J.Phys.Chem.Solids 46, 331 (1985)

[8] H. Liu, W. A. Caldwell, L. R. Benedetti, W. Panero, and R. Jeanloz, Phys. Chem. Miner. 30, 582 (2003).

[9] M.P. Pasternak, G.Kh. Rozenberg, G.Yu. Machavariani, O. Naaman, R.D. Taylor, and R. Jeanloz, Phys. Rev. Lett. 82, 4663 (1999).

[10] G.Kh. Rozenberg, L.S. Dubrovinsky, M.P. Pasternak, O. Naaman, T. Le Bihan, and R. Ahuja, Phys. Rev. B 65, 064112 (2002).

[11] J. Badro, G. Fiquet, V.V. Struzhkin, M. Somayazulu, H.K. Mao, G. Shen, and T. Le Bihan, Phys. Rev. Lett. 89, 205504 (2002).

[12] L. M. Sandratskii, M. Uhl, and J. Kübler, J. Phys.: Condens. Matter 8, 983 (1996).

[13] G. Rollmann, A. Rohrbach, P. Entel, and J. Hafner, Phys. Rev. B 69, 165107 (2004); M. P. J. Punkkinen, K. Kokko, W. Hergert, and J. J. Väyrynen, J. Phys.: Condens. Matter 11, 2341 (1999); A. Bandyopadhyay, J. Velev, W. H. Butler, S.K. Sarker and O. Bengone, Phys. Rev. B 69, 174429 (2004); V. V. Mazurenko and V. I. Anisimov, Phys. Rev. B 71, 184434 (2005).

[14] J. Kuneš, A. V. Lukoyanov, V. I. Anisimov, R. T. Scalettar, and W. E. Pickett, Nature Materials 7, 198 (2008).

[15] A. V. Kozhevnikov, A. V. Lukoyanov, V. I. Anisimov, and M. A. Korotin, J. Exp. Theor. Phys. 105, 1035 (2007).

[16] J. Kuneš, V. I. Anisimov, A. V. Lukoyanov, and D. Vollhardt, Phys. Rev. B 75, 165115 (2007).

[17] Dm. Korotin, A. V. Kozhevnikov, S. L. Skornyakov, I. Leonov, N. Binggeli, V. I. Anisimov, and G. Trimarchi, Eur. Phys. J. B 65, 91 (2008); S. Baroni, A. Dal Corso, 
S. de Gironcoli, P. Giannozzi, C. Cavazzoni, G. Ballabio, S. Scandolo, G. Chiarotti, P. Focher, A. Pasquarello, K. Laasonen, A. Trave, R. Car, N. Marzari, and A. Kokalj, http://www.pwscf.org/

[18] V.I. Anisimov, J. Zaanen and O.K. Andersen, Phys. Rev. B 44, 943 (1991).

[19] A. E. Bocquet, T. Mizokawa, T. Saitoh, H. Namatame and A. Fujimori, Phys. Rev. B 46, 3771 (1992).
[20] P. Werner, A. Comanac, L. de Medici, M. Troyer, and A. J. Millis, Phys. Rev. Lett. 97, 076405 (2006).

[21] P. Werner and A. J. Millis, Phys. Rev. Lett. 99, 126405 (2007).

[22] F. Ciccacci, L. Braicovich, E. Puppin, and E. Vescovo, Phys. Rev. B 44, 10444 (1991). 